\title{
Performance Evaluation of Fuzzy Ant Based Routing Method for Connectionless Networks
}

\author{
Seyed Javad Mirabedini ${ }^{1}$ and Mohammad Teshnehlab ${ }^{2}$ \\ ${ }^{1} \mathrm{Ph} . \mathrm{D}$. Student of Computer Software, Tehran Markaz Branch of \\ Islamic Azad University, Tehran, Iran \\ jvd2205@yahoo.com \\ ${ }^{2}$ Electrical Eng.K.N.Tossi University, Tehran, Iran \\ teshnehlab@eetd.kntu.ac.ir
}

\begin{abstract}
This paper introduces a novel algorithm, called FuzzyAntNet inspired by swarm intelligence and optimized by fuzzy systems. FuzzyAntNet is a new routing algorithm which is constructed by the communication model observed in ant colonies, and enhanced with fuzzy systems. Two special characteristics of this method are scalability to network changes and capability to recognize the best route from source to destination with low delay, traffic and high bandwidth. Using Ants (or agents) in this method cause to avoid congestion in data packet transmission because Ants walk through paths and intermediate routers and gather information about their delay, congestion and in return, they update the current delay of each visited link and consequently they bring up to date the routing probabilities table for every traversed router. We compare FuzzyAntNet with other routing algorithms such as AntNet and Destination Sequenced Distance Vector (DSDV).
\end{abstract}

Keywords: Connectionless Networks, Routing Algorithms, Ant Colony System, Fuzzy Systems, Fuzzy Ant Based Routing, Probabilities Table, Fuzzydelay.

\section{Introduction}

Routing algorithms in modern networks must address numerous problems. Current routing algorithms are not adequate to tackle the increasing complexity of such networks. Centralized algorithms have scalability problems; static algorithms have trouble keeping up-to-date with network changes; and other distributed and dynamic algorithms have oscillations and stability problems. One of the applications of Ant Colony Optimization in network routing is swarm intelligence routing which provides a promising alternative to these approaches. Swarm intelligence utilizes mobile software agents for network management. These agents are autonomous entities, both proactive and reactive, and have the capability to adapt, cooperate and move intelligently from one location to the other in the communication network. In this paper we discuss routing algorithms in section 2, present FuzzyAntNet in section 4, and finally the simulation and results are given in section 5. 


\section{Routing Algorithms}

Routing decisions can only be made on the basis of local and approximate information about the current and the future network states, with additional constraints posed by the network switching and transmission technology [1]. In this article we focus on wide area networks, that is, irregular topology datagram networks with an IP-like (Internet Protocol) network layer and a very simple transport layer. The instance of the communication network is mapped on a directed weighted graph with $N$ nodes. All the links are viewed as having two parameters characterized by a bandwidth and a transmission delay, and are accessed following a statistical multiplexing scheme.

\section{Ant Based Routing}

In the Ant-based routing algorithm, routing is determined by means of very complex interactions of forward and backward network exploration ants. The idea behind this subdivision of agents is to allow the backward ants to utilize the useful information gathered by the forward ants on their trip from source to destination. Based on this principle, no node routing updates are performed by the forward ants. The backward ants inherit this raw data and use it to update the routing table of the nodes [2], [3].

Table 1. Ant - Based Routing Table for Node A

\begin{tabular}{|l|c|c|c|}
\hline \multirow{3}{*}{ Node A } & \multirow{2}{*}{ Destination } & \multicolumn{2}{|c|}{ Neighbor Node } \\
\cline { 2 - 4 } & & $\mathbf{B}$ & $\mathbf{C}$ \\
\cline { 2 - 4 } & $\mathbf{E}$ & $\mathbf{0 . 3 5}$ & $\mathbf{0 . 6 5}$ \\
\cline { 2 - 4 } & $\mathbf{F}$ & $\mathbf{0 4 0}$ & $\mathbf{0 . 6 0}$ \\
\hline
\end{tabular}

In table I, a probability value $P_{d n}$ which expresses the probability of choosing $n$ as neighbor node when the destination node is $d$, with the constraint defined in (1):

$$
\sum_{n \in N_{k}} P_{d n}=1 \quad, d \in[1, N] \quad, N_{k}=\{\text { neighbor' } s(k)\} .
$$

\section{Fuzzy Ant Based Routing Method}

In this section we describe our novel algorithm which we called it FuzzyAntNet. FuzzyAntNet is constructed by the stigmergy communication model observed in ant colonies and applied with fuzzy systems [4], [5], [6]. In this algorithm every link between two nodes $\mathrm{i}$ and $\mathrm{j}$ is addressed as $\operatorname{link}_{\mathrm{ij}}$ and there are two parameters for each $\operatorname{link}_{\mathrm{ij}}$ : Delay $\mathrm{y}_{\mathrm{ij}}$ and Bandwidth $_{\mathrm{ij}}$. There are five membership functions for first input variable $\left(\right.$ Delay $\left._{\mathrm{ij}}\right)$, five membership functions for second input variable (Bandwidth ${ }_{\mathrm{ij}}$ ), and nine membership functions for output variable (Fuzzy-Delay ${ }_{\mathrm{ij}}$ ). All of the membership functions are Triangular because it can eliminate noises and in comparison with Gaussian membership function, the precision of Triangular is as good as Guassian 
but it's computation is so easier and simpler. Before applying their values, Delay ${ }_{\mathrm{ij}}$ is normalized between $(0,1)$ and Bandwidth $_{\mathrm{ij}}$ is normalized between $(0,0.5)$. The engine used, is multiplication engine. Table 2 shows the rulebase for the fuzzy system. In this table the Values for the amount of goodness from lowest to highest are LL(Very Low), LM, LH, ML, MM, MH, HL, HM, and HH(Very High).

Table 2. RuleBase for FuzzyAntNet Method

\begin{tabular}{|l|c|c|c|c|c|}
\hline $\begin{array}{c}\text { X1 } \\
\text { X2 (Delay) } \\
\text { (Bandwidth) }\end{array}$ & $\begin{array}{c}\text { VL } \\
\text { (Very Low) }\end{array}$ & $\begin{array}{c}\text { L } \\
\text { (Low) }\end{array}$ & $\begin{array}{c}\text { M } \\
\text { (Medium) }\end{array}$ & $\begin{array}{c}\mathrm{H} \\
\text { (High) }\end{array}$ & $\begin{array}{c}\text { VH } \\
\text { (Very High) }\end{array}$ \\
\hline VL (Very Low) & MM & ML & LH & LM & LL \\
\hline L (Low) & MH & MM & ML & LH & LM \\
\hline M (Medium) & HL & MH & MM & ML & LH \\
\hline H (High) & HM & HL & MH & MM & ML \\
\hline VH (Very High) & HH & HM & HL & MH & MM \\
\hline
\end{tabular}

In table 2 as shown, there are 25 rules for this fuzzy system. We mentioned some of them as following:

$\mathrm{R}_{1}$ : If $\mathrm{x} 1$ (Delay) is $\mathrm{VL}$ and $\mathrm{x} 2$ (bandwidth) is $\mathrm{VL}$ then $\mathrm{Y}$ (goodness ) is MM $\mathrm{R}_{25}$ If $\mathrm{x}$ 1(Delay) is $\mathrm{VH}$ and $\mathrm{x} 2$ (bandwidth) is $\mathrm{VH}$ then $\mathrm{y}$ (goodness ) is $\mathrm{MM}$

The output of fuzzy system is named Fuzzy_Delay ${ }^{\mathrm{k}}{ }_{\mathrm{ij}}(\mathrm{t})$. In simulation it is replaced with delay $y_{\mathrm{ij}}$ and computed by (2):

$$
\text { Fuzzy _ Delay }{ }_{i j}(t)=\frac{\sum_{l=1}^{M} y \prod_{i=1}^{-n_{f}} \mu_{A_{i}^{l}\left(x_{i}\right)}}{\sum_{l=1}^{M} \prod_{i=1}^{n_{f}} \mu_{A_{i}^{l}\left(x_{i}\right)}}
$$

where the parameters are:

$\mathrm{i}$ : the node where an ant is going from .

$\mathrm{j}$ : the node where an ant wants to move.

$\mathrm{M}$ : the number of fuzzy rule bases used $(\mathrm{M}=25)$.

$\mathrm{n}_{\mathrm{f}}$ : the number of membership functions for input variables $\left(\mathrm{n}_{\mathrm{f}}=2\right)$.

$\mu_{\mathrm{Ai}(\mathrm{xi})}^{1}$ : The Fuzzy value of membership functions.

In FuzzyAntNet nodes launch forward ants in regular intervals. The forward ant keeps track of the visited notes in a stack $J^{k}$ and of their associated cost Fuzzy _ Delay ${ }_{j, d}^{n}$. This cost can be the wait time in queue and the transmission delay for each visited node $n$. The cost Sum $m_{-} F u z z y_{-}$Delay $_{j, d}^{n}$ is defined as the sum of all the delay costs from node $n$ to destination node $d$. Once the destination $d$ is reached, 
then a backward ant is launched, which updates the distance estimation Sum_Fuzzy _ Delay ${ }_{j, d}^{n}$ for node $n$ to $d$ via $j$ as shown in (3):

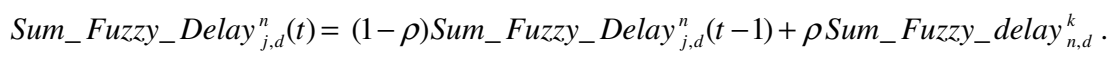

Where $\rho$ is the learning rate and set to 0.7 . The routing table probabilities, are updated by (4):

$$
P_{j, d}^{n}(t)=\frac{\left[\begin{array}{c}
1 \\
\text { Sum_Fuzzy Delay }{ }_{j, d}^{n}(t)
\end{array}\right]^{\beta}}{\sum_{l}\left[\frac{1}{\text { Sum_Fuzzy _ Delay }{ }_{l, d}^{n}(t)}\right]^{\beta}}
$$

Where $l \in \operatorname{Neighbor}(n)$ and $\beta$ is a non-linearity factor .In this experiment $\beta$ is set to 1 .

\section{Simulation and Results}

In our experiments, we compared FuzzyAntNet to a set of state-of-the-art algorithms in a realistic network simulator which constructed by object oriented programming in $\mathrm{C}++$ and used network simulator 2 (ns2) as Constant Bit Rate (CBR) traffic generator. Both data packets and ants packet have 512 bytes length. The rate of ant packet (overhead) for both AntNet and FuzzyAntNet is only one percent. We used a typical network topology called TypicalNet as shown in Fig. 1.

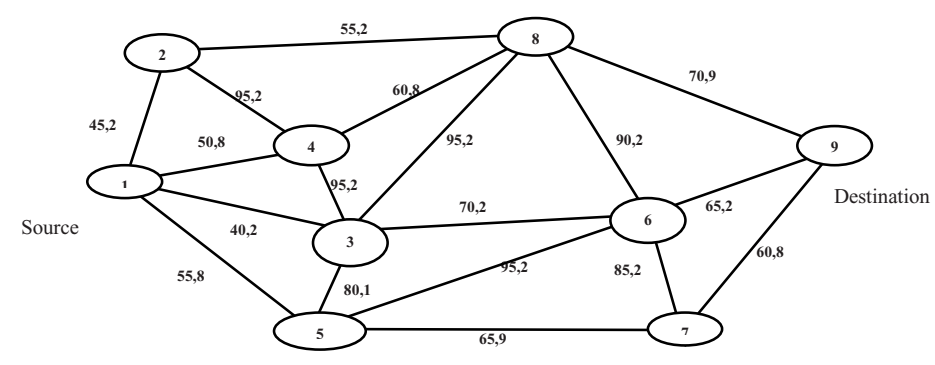

Fig. 1. TypicalNet. On every link, it's delay and bandwidth are shown respectively.

In TypicalNet, the the traffic is generated by source node 1 and the destination is node 9. Standard performance metrics are: Throughput, End-2-End Delay, Packet Delivery, and Packet Drop Ratio. Comparisons of End-2-End Delay are performed for DSDV, AntNet, and FuzzyAntNet and the results are shown in Fig. 2.

We also summarized the experimental results in Table 3 which shows that FuzzyAntNet outperforms other routing methods in all evaluation metrics. 


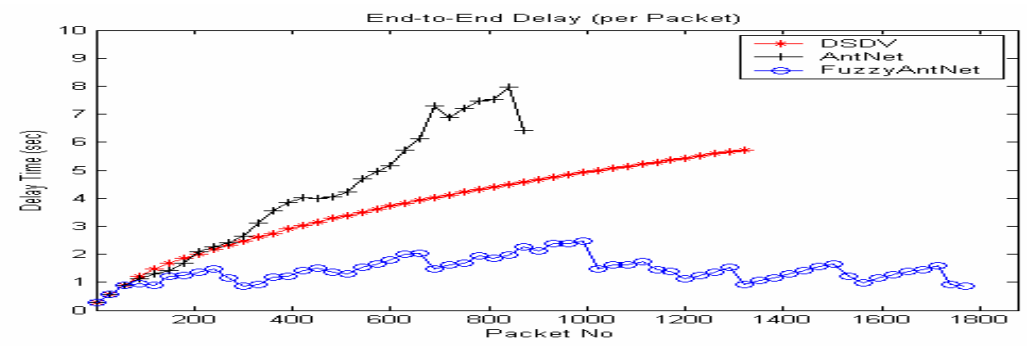

Fig. 2. Simulation results for End-2-End delay. In this software-simulated network during 20 seconds, the routing algorithms have been executed in the same traffic state.

Table 3. Results obtained by three algorithms DSDV, AntNet, and FuzzyAntNet

\begin{tabular}{|c|c|c|c|}
\hline \multirow{2}{*}{$\begin{array}{c}\text { Routing } \\
\text { Standard } \\
\text { Criteria }\end{array}$} & DSDorithms & AntNet & FuzzyAntNet \\
\cline { 2 - 4 } & Overhead(\%0) & Overhead(\%1) & Overhead(\%1) \\
\hline Avg. End-to-End Delay (s) & 4.43 & 4.36 & $\mathbf{2 . 9 9}$ \\
\hline Avg.Throughput (kbps) & 280.8 & 189.2 & $\mathbf{3 7 5}$ \\
\hline Packet Delivery Ratio (\%) & 70 & 47 & $\mathbf{9 3}$ \\
\hline Packet Drop Ratio (\%) & 30 & 53 & $\mathbf{7}$ \\
\hline
\end{tabular}

\section{Conclusions}

In this paper we introduced a novel method called FuzzyAntNet which showed a scalable and robust mechanism with the ability to reach a stable behavior even in changing network envornment. FuzzyAntNet is outperformed in all metrics in this simulation. It also expresses good utilization of network, balancing the data packets through networks which reduces congestion and avoids packet drops.

\section{References}

1. Bertsekas D.\&Gallager R.: Data Networks. Englewood Cliffs, Prentice-Hall (1992)

2. Di Caro G.\& M.Dorigo: Ant Colonies for Adaptive Routing in Packet Switched Comunications Networks. Proc. PPSN Fifth International Conference on Parallel Problem Solving From Nature (1998)

3. G. Di Caro and M. Dorigo: "AntNet: A Mobile Agents Approach to Adaptive Routing", Tech. Rep. IRIDIA/97, Université Libre de Bruxelles, Belgium (1997)

4. Dubois, D. and H. Prade: Fuzzy Sets and Systems: Theory and Applications, Vol.18 Filev, D.P. (1996) 
5. Seyed Javad Mirabedini, Mohammad Teshnehlab: "Adaptive Neuro Fuzzy for Optimization of Ant Colony System". Workshop Proc. EurAsia-ICT Advances in Infor-mation and Communication Technology, Shiraz Iran (2002) 325-329

6. Seyed Javad Mirabedini , Mohammad Teshnehlab: "AntNeuroFuzzy: Optimal Solu-tion for Traveling Salesman Problem using Ant Colony and Neuro-Fuzzy Systems".Proc. ICTIT International Conference Supported by IEEE Jordan (2004) 305-312 